\title{
Late Onset Horner's Syndrome during Postoperative Epidural Analgesia
}

\author{
Kanchan Rani, Mukesh Kumar Prasad \\ Dept. of Obs /Gyn, Government Medical College, Nainital, Uttranchal, India
}

\section{To the Editor,}

Horner's syndrome though uncommon ${ }^{1}$, is a welldocumented complication of epidural analgesia ${ }^{2}$.Here we report a case of Horner's syndrome developed 24 hours after starting of epidural analgesia during post operative period following emergency caesarean section.

A 24 year primigravida at term pregnancy presented in Emergency Department with labor pains and history of leaking per vaginum. On examination cervix was $2 \mathrm{~cm}$ dilated, $80 \%$ effaced with vertex presentation. Leaking was present with meconium staining of liqor. Fetal heart rate was in range of 100-106 beats per minute. Emergency caesarean section was planned under general anesthesia followed by epidural catheter placement for post operative analgesia. Caesarean section was uneventful and then she was reversed from general anesthesia. 20-gauge epidural catheter was placed at T6-7 space in lateral position. She received initial bolus dose of $10 \mathrm{ml} 0.2 \%$ Ropivacaine with 50 microg fentanyl followed by epidural catheter infusion of $0.2 \%$ and $0.001 \%$ fentanyl at a rate of $10 \mathrm{ml} / \mathrm{h}$. The patient was pain free till 24 hours. After 24 hours patient started complaining of pain and heaviness in right eye. Examination revealed contracted pupil, a drooping eyelid, and conjunctival injection, accompanied by flushing on the same side of the face. Reduced sensation over right hemifacial region and upper arm was noted. Loss of suckling sensation (T4) along with partial numbness was elicited over right upper arm. A diagnosis of right Horner's syndrome was made. Ropivacaine fentanyl infusion was stopped. Signs resolved over the next 2 hour without treatment.
Analgesia thereafter was maintained by intravenous PCA morphine and NSAID.

Horner's syndrome in most of the earlier reported cases appeared within 24 hours and disappeared on discontinuation of infusion. Late onset may have resulted due to asymmetrical positioning of catheter in extradural space, partial migration of epidural catheter in subdural space or anatomical changes in extradural space of a pregnant women ${ }^{3,4}$.Early recognition of transient Horner's syndrome prevents unnecessary anxiety and potential initiation of unnecessary diagnostic workup, given that the clinical course is favorable with spontaneous resolution.

\section{References}

1. Mohan J, Potter JM. Pupillary constriction and ptosis following caudal epidural analgesia. Anaesthesia 1975; 30: 769-73.

2. Clayton KC.The incidence of Horner's syndrome during lumbar extradural for elective Caesarean section and provision of analgesia labour. Anesthesia1983;38:583-5.

3. Dela GF, Reyes A, Avellanal M, Baticon P, Gonzalez-Zarco LM. Trigeminal nerve palsy and Horner's syndrome following epidural analgesia for labor: a subdural block. Int J Obstet Anesth $2007 ; 16: 180-2$.

4. Narouze SN,Basali A, Mandel M, Tetzlaff JE.Horner's syndrome and trigeminal nerve palsy after lumbar epidural analgesia for labor and delivery. J Clin Anesth 2002;14:532-4. 